\title{
A novel method to prevent control system instability based on a soft computing knowledge system
}

\author{
José Luis Calvo-Rolle ${ }^{1}$ Emilio Corchado², Ramón Ferreiro ${ }^{1}$, Amer Laham², \\ $M^{a}$ Araceli Sánchez ${ }^{2}$, Ana Gil ${ }^{2}$
}

\begin{abstract}
The aim of this study is to present a novel soft computing method to assure PID tuning parameters place the system into a stable region by applying the gain scheduling method. First the system is identified for each significant operation point. Then using transfer functions solid structures of stability are calculated to program artificial neural networks, whose object is to prevent system from transitioning to instability. The method is verified empirically under a data set obtained by a pilot plant.
\end{abstract}

Keywords: KBS, Robust stability, artificial neural networks, soft computing.

\section{Introduction}

Continuous research is necessary in the field of process engineering to define new methods of regulation, in order to improve current ones [1]. The demand for system control applications is driven by the increasingly numerous ranges of possibilities [2, 3] that are nowadays in use or under development.

This research introduces an artificial neural network (ANN) to prevent control system instability that is regulated using gain scheduling with predetermined PID (Proportional-Integral-Derivative) coefficients. The method is validated on a laboratory stove. Nevertheless, the vast majority, as many as $90 \%$ [4], of control loop systems use PID controllers.

When dealing with non-liner systems, certain specifications have to be equal in all areas of operation. The regulator will therefore require different parameters for each area. These problems can be reduced by using self-regulating and adaptive PID controllers [5]. It should be noted, however, that their implementation can be expensive and specific to the type of process that it is meant to regulate, which further complicates any general theory on PID controllers. Many of the drawbacks resulting from self-regulating and adaptive PID controllers are alleviated using the

\footnotetext{
${ }^{1}$ University of Coruña, Department of Industrial Engineering Avda. 19 de febrero, s/n, 15405, Ferrol, A Coruña, Spain \{jlcalvo, ferreiro\}@cdf.udc.es

${ }^{2}$ University of Salamanca, Departmento de Informática y Automática Plaza de la Merced s/n, 37008, Salamanca, Spain \{escorchado, maraceli, abg\}@usal.es
} 
well-known Gain Scheduling method [6] considered as part of the family of adaptive controllers [5]. Significant system variables that define the point of operation have to be selected in order to implement the Gain Scheduling method. It is then necessary to choose several regions throughout the entire operating range of the plant, in which the behaviour is linear. The controller parameters are then fixed which provide similar specifications for the operating range of the plant.

The system may be stable for controller parameters that are deduced, but it may not be stable between the selected points or regions. There is no simple solution to this situation, which is usually broken down into constituent parts. This is the reason to propose a Knowledge Base System (KBS) [7] as a possible solution.

The Gain Scheduling method selects the correct controller parameters, though operators often adjust the parameter values with the aim of improving plant specifications. Sometimes, the parameters they assign may cause instability. The novel soft computing method proposed in this research is intended to prevent instability. Artificial neural networks are proposed as means of overcoming the problem [9]. Essentially this method decides whether PID parameters programmed by human operators are valid and whenever the plant to control enters an unstable zone due to parameter combinations, the method restores a more stable combination for the operation point in question.

\section{A Novel Soft computing controller topology}

The proposed topology is based on traditional PID. There are many ways to represent PID controllers, but perhaps the most widely used is given in equation 1, [4].

$u(t)=K\left[e(t)+\frac{1}{T_{i}} \int_{0}^{t} e(t) d t+T_{d} \frac{d e(t)}{d t}\right]$

Where ' $u$ ' is the control variable and ' $e$ ' is the control error given by ' $e=S P$ $y$ ' (difference between ' $S P$ ', the reference specified by the input and ' $y$ ', the measurement unit specified by the output). The other terms are the tuning controller parameters: proportional gain ' $K$ ', integral gain ' $T i$ ' and derivate gain ' $T d$ '.

System dynamics change with process operation conditions. Changes in a dynamic process may, for instance, be caused by well-known nonlinearities inherent in the system. It is possible to modify the control parameters, by monitoring their operating conditions and establishing rules. The methodology comprises the following steps: first of all, Gain Scheduling is applied, then the behaviour of the plant to control is analyzed at different points of interest, and, finally, rules are established to program gains in the controller. It would then be possible to obtain certain specifications which remain constant throughout the whole range of operation. In the proposed method, it is possible to change the PID parameter values to improve the operating conditions, but the possibility of undesirable parameter combinations must be prevented. This idea is schematically represented in Figure 1. 
Fig. 1. Gain Scheduling with proposed topology.

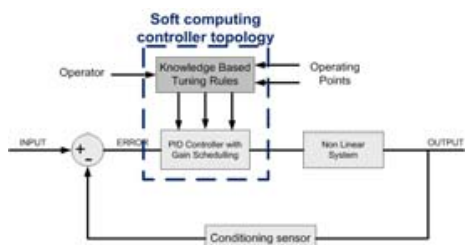

The idea of Gain Scheduling is to obtain the PID parameters when given the operating points. In this case, a new input has been added, with which the operator can modify the other parameters taken from knowledge based tuning rules. Figure 2 shows a basic diagram of the suggested structure for the method in which PID parameters may be adjusted by the operator. If the input causes system instability, the proposed Soft computing topology can commute parameters and program the controller to maintain it within the dynamic range of the plant.

There is an artificial MLP-type neural network [10] in the proposed system, which must be trained to detect the existence of stable parameter combinations. In case of instability, the system will revert back to stable values according to the operating work point that was initially programmed.

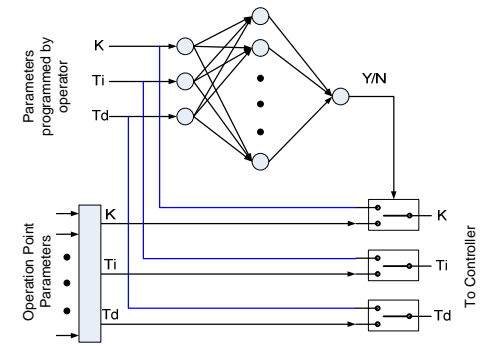

Fig. 2. Multi-Layer Perceptron Neural Network architecture.

In order to apply a Multi-Layer Perceptron (MLP) network, an interesting and informative data set must be chosen. To that end, solid stability and instability structures were applied, in order to delimit both states in absolute terms. These structures are defined by PID controller parameters along with their stability/instability (both states do not coexist) points that have to be placed into three axes of a threedimensional graphic. Abundant literature exists on robust stability problems that describe this concept $[11,12]$. The volume consists of parameter combinations $(K$, $T i$ and $T d$ ) of the controller for a stable system. If the structure was unstable, then the volume would consist of the parametric controller combinations for an unstable system.

\section{A case study: empirical verification of the proposed method}

An empirical verification of the proposed novel method was performed on a small pilot plant (figure 3 ) in which the temperature is controlled. Temperature depends on the following parameters: $\mathrm{T}_{1}(\mathrm{t})$ is the temperature measure outside the stove, $\mathrm{V}$ is the air volume in the stove, $\operatorname{SP}(\mathrm{t})$ is the set point for the desired temperature, 
$\mathrm{T}_{2}(\mathrm{t})$ is the measure temperature in the recipient, $\mathrm{u}(\mathrm{t})$ is the signal control to operate the heating element, $\mathrm{Kv}$ and $\mathrm{Kt}$ are constants relating to features of the heating element properties and the temperature sensor respectively.
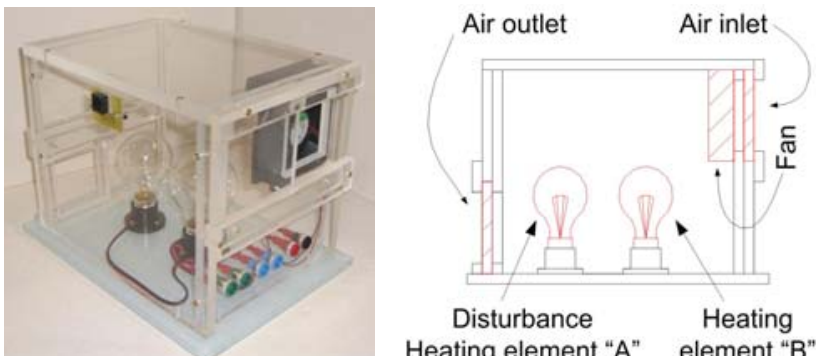

Fig. 3. Photograph and schematic diagram of the real plant.

The pilot plant used for these experiments consists of a stove, with an air stream (Fig.3), which will be controlled to maintain a constant temperature inside. It is a non-linear system among others due to heating element.

The regulator is a virtual controller that takes signals from the plant through a data acquisition card, which is fed into the Simulink Matlab software. As a set point signal, the plant receives the required temperature inside and adjusts the power applied to regulate the input flow into the tank.

\subsection{System operation conditions which define a Soft Computing Knowledge Base System (KBS)}

The operation conditions of the system are infinite; certain values must therefore be chosen. Coherent estimates values are necessary to achieve good results and it makes no sense to obtain parameters for multiple cases. One approach is to choose a reasonable amount of equidistant values and observe the parametrical changes in each case. An opportunity arises to define new intermediate values if there are substantial changes from one value to another.

Certain characteristics of the stove such as its air volume remain constant. In this case the only term that defines the operation conditions or gains adjustment rules of the controller is the outside temperature. It should be highlighted that certain changes may occur under field conditions, such as variations in pressure, noisier communications, dirty system components, humidity, distance between control and actuators or sensors. Taking into account the pilot plant and the value ranges that may be taken from the temperature inside, ten different operating conditions (ranges) were established: $\left(10^{\circ} \mathrm{C}-15^{\circ} \mathrm{C}\right),\left(15^{\circ} \mathrm{C}-20^{\circ} \mathrm{C}\right), \ldots,\left(55^{\circ} \mathrm{C}-60{ }^{\circ} \mathrm{C}\right)$. As it will be seen in the final results, the chosen range of temperatures filling values will be sufficient to cover the entire operating range of the system.

\subsection{Obtaining the controller parameters for each operation point}

A hysteresis block could be selected to obtain the regulator parameters of the different working points, in parallel with the PID controller, before applying the Relay Feedback method. 
The Relay Feedback method is an alternative to the Ziegler-Nichols closed loop [13], for the empirical location of the critical gain (Kc) and the period of sustained oscillation (Tc) of the system. The method, developed by Aström and Hägglud [5], fixed the system in its oscillation state. Its implementation scheme is shown in Figure 4. The Relay Feedback has the advantage that an adjustment can be made to the set point at any time.

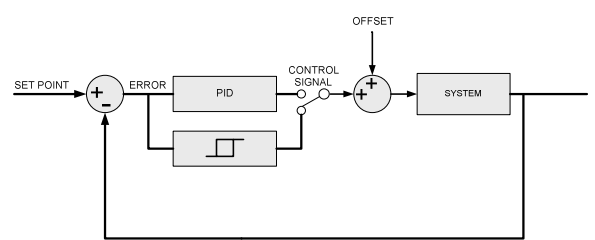

Fig. 4. Diagram of Relay-Feedback with relay and PID controller option.

This system oscillation has a period with approximately the same value as the period in the Ziegler-Nichols method. A relay with hysteresis centered on the zero value with an amplitude $d$ and a hysteresis window width of $h$ is recommended for the general method.

\subsection{Procedures carried out}

Obtaining certain parameters: $\mathbf{T}_{\mathbf{c}}$ and $\mathbf{K}_{\mathbf{c}}$. As this particular case study is working with a slow system, there is no need to implement the hysteresis cycle mentioned in the above explanation of the Relay Feedback with a window. Instead, a simple comparator is enough ( $h=0$ and $d=0.5$ ). The offset for this case is not necessary because it would be zero. When the system is in operation and sufficient time has elapsed, oscillation is stabilized and periodic. It is then necessary to pay attention to the final zone, and with the expressions for the Relay Feedback method, the extracted parameters are Tc and Kc.

Obtaining the initial PID controller parameters. With the parameters that have been obtained in the previous step, it is possible to get the controller parameters to apply direct formulas, thereby achieving the three terms of the regulator: $(\mathrm{K}, \mathrm{Ti}$ and Td). In this system, they will have to be obtained from load disturbance criterion, after which the Ziegler-Nichols closed loop method is applied [4].

Controller fine tuning. When the results are unsatisfactory, subsequent manual fine-tuning may occasionally be needed after having obtained the parameters with the relay feedback method. This is a delicate adjustment which should not at any point saturate the output controller. A compromise has to be found, without the proportional component $(\mathrm{K})$ being excessive, that would cause a rapid response in the output and little overoscillation, which would apparently be ideal. However, under these conditions the heating element will be in a state of constant power, which will lead to its deterioration within a short period of time. In conclusion, it is necessary to search for gradual outputs, without saturation or sudden changes. Obtaining the parameters for each case, taking into account all the aspects commented on above, the criteria for the fine-tuned controller parameters in each case are minimum overoscillation and maximum speed for the restrictions presented in the preceding paragraphs. So, this leads to the parameters shown in Table 1. 
Table 1. PID parameters values for each operating range.

\begin{tabular}{|c|c|c|c|c|c|c|c|}
\hline Level & $\mathbf{K}$ & $\mathbf{T i}$ & Td & Level & $\mathbf{K}$ & $\mathbf{T i}$ & Td \\
\hline $10 C^{0}-15 C^{\circ}$ & 8.1 & 18 & 4.2 & $35 C^{\circ}-40 C^{\circ}$ & 7.5 & 21 & 4.2 \\
\hline $15 C^{0}-20 C^{\circ}$ & 8.5 & 21 & 4.3 & $40 C^{0}-45 C^{\circ}$ & 7.9 & 21 & 4.3 \\
\hline $20 C^{\circ}-25 C^{\circ}$ & 8.0 & 19 & 4.5 & $45 C^{\circ}-50 C^{\circ}$ & 7.7 & 20 & 4.3 \\
\hline $25 C^{0}-30 C^{\circ}$ & 8.1 & 17 & 4.3 & $50 C^{\circ}-55 C^{\circ}$ & 7.4 & 19 & 4.0 \\
\hline $30 C^{\circ}-35 C^{\circ}$ & 8.9 & 18 & 4.7 & $55 C^{\circ}-60 C^{\circ}$ & 7.1 & 19 & 4.1 \\
\hline
\end{tabular}

Neural Network implementation. Different Multi-Layer Perceptron (MLP) [8] network was applied for each operation condition range in this research, in order to detect parameter values that lead to system instability. Firstly, it was necessary to obtain the transfer function for each operating point under consideration. To do so, the system identification was obtained at each point by applying an ARX (Auto-Regressive models with eXogenous inputs) method [14] using the Matlab Identification Toolbox. Other identification methods used were AR (AutoRegressive model) and ARMAX (AutoRegressive Moving Average model with eXogenous inputs model) but the best results were achieved with ARX for the plant used in the experiment (Table 2 shows the Fit between the real plant and the model, and the final prediction error (FPE) that provides a measure of model quality).

Stability structures were then obtained, and the Neural Network architecture was trained with those same points. A neural network was obtained for all operating points. The number of neurons in its intermediate layer fluctuated between 7 and 10 . The structure was adopted after rigorous testing with different numbers of neurons in the hidden layer (tests were conducted with 5 to 12 neurons in the intermediate layer) for every neural network.

The activation transfer functions of the hidden layer are hyperbolic tangent types. Other activation functions were probed, although the best results (best error percentage) for each operation point in all the ANNs were achieved with the hyperbolic tangent function (Table 3). In the output layer, a hard limit transfer function was applied to indicate whether the parameters programmed by human operator are valid or whether it is necessary to apply corresponding stored parameters to the operation point.

Table 2. Comparison of identification method parameters (Fit and Final Prediction Error (FPE)).

\begin{tabular}{|c|c|c|c|c|c|c|}
\hline \multirow{2}{*}{$\begin{array}{l}\text { Transfer func- } \\
\text { tion range }\end{array}$} & \multicolumn{2}{|c|}{ AR } & \multicolumn{2}{|c|}{ ARX } & \multicolumn{2}{|c|}{ ARMAX } \\
\hline & Fit(\%) & FPE & Fit(\%) & FPE & Fit(\%) & FPE \\
\hline $10 C^{o}-15 C^{o}$ & 43.2 & $>10 \mathrm{e}-6$ & 74.2 & $<10 \mathrm{e}-6$ & 60.1 & $<10 \mathrm{e}-6$ \\
\hline $15 C^{\circ}-20 C^{o}$ & 46.2 & $>10 \mathrm{e}-6$ & 75.1 & $<10 \mathrm{e}-6$ & 61.2 & $<10 \mathrm{e}-6$ \\
\hline $20 C^{\circ}-25 C^{\circ}$ & 48.3 & $>10 \mathrm{e}-6$ & 75.0 & $<10 \mathrm{e}-6$ & 62.4 & $<10 \mathrm{e}-6$ \\
\hline $25 C^{\circ}-30 C^{\circ}$ & 45.7 & $>10 \mathrm{e}-6$ & 74.7 & $<10 \mathrm{e}-6$ & 63.2 & $<10 \mathrm{e}-6$ \\
\hline $30 C^{\circ}-35 C^{\circ}$ & 47.7 & $>10 \mathrm{e}-6$ & 75.3 & $<10 \mathrm{e}-6$ & 63.8 & $<10 \mathrm{e}-6$ \\
\hline $35 C^{\circ}-40 C^{\circ}$ & 47.3 & $>10 \mathrm{e}-6$ & 76.6 & $<10 \mathrm{e}-6$ & 63.1 & $<10 \mathrm{e}-6$ \\
\hline $40 C^{\circ}-45 C^{\circ}$ & 46.2 & $>10 \mathrm{e}-6$ & 76.1 & $<10 \mathrm{e}-6$ & 64.4 & $<10 \mathrm{e}-6$ \\
\hline $45 C^{\circ}-50 C^{\circ}$ & 49.9 & $>10 \mathrm{e}-6$ & 77.5 & $<10 \mathrm{e}-6$ & 65.1 & $<10 \mathrm{e}-6$ \\
\hline $50 C^{\circ}-55 C^{\circ}$ & 50.1 & $>10 \mathrm{e}-6$ & 76.5 & $<10 \mathrm{e}-6$ & 61.2 & $<10 \mathrm{e}-6$ \\
\hline $55 C^{\circ}-60 C^{\circ}$ & 52.2 & $>10 \mathrm{e}-6$ & 77.6 & $<10 \mathrm{e}-6$ & 62.1 & $<10 \mathrm{e}-6$ \\
\hline
\end{tabular}


Table 3. Comparison between ANN activation transfer functions (Linear, Log-Sigmoid, Tan-Sigmoid) in hidden layer (best error (E\%) and number of neurons in middle layer $(\mathrm{Ne})$ ).

\begin{tabular}{|c|c|c|c|c|c|c|c|c|c|c|c|c|c|}
\hline \multirow{2}{*}{ ANN range } & \multicolumn{2}{|c|}{ linear } & \multicolumn{2}{|c|}{ logsig } & \multicolumn{2}{|c|}{ tansig } & \multirow{2}{*}{ ANN range } & \multicolumn{2}{|c|}{ linear } & \multicolumn{2}{|c|}{ logsig } & \multicolumn{2}{|c|}{ tansig } \\
\hline & E\% & $\mathrm{Ne}$ & E\% & $\mathrm{Ne}$ & E\% & $\mathrm{Ne}$ & & E\% & $\mathrm{Ne}$ & E\% & $\mathrm{Ne}$ & E\% & $\mathrm{Ne}$ \\
\hline $10 C^{0}-15 C^{0}$ & 18 & 7 & 10 & 6 & 2 & 7 & $35 C^{\circ}-40 C^{o}$ & 20 & 8 & 13 & 7 & 0 & 8 \\
\hline $15 C^{0}-20 C^{0}$ & 20 & 8 & 11 & 7 & 2 & 7 & $40 C^{\circ}-45 C^{\circ}$ & 19 & 8 & 11 & 7 & 1 & 9 \\
\hline $20 C^{\circ}-25 C^{\circ}$ & 21 & 8 & 13 & 6 & 1 & 8 & $45 C^{\circ}-50 C^{\circ}$ & 19 & 7 & 16 & 8 & 1 & 9 \\
\hline $25 C^{\circ}-30 C^{\circ}$ & 22 & 7 & 15 & 6 & 0 & 8 & $50 C^{\circ}-55 C^{\circ}$ & 21 & 7 & 14 & 8 & 0 & 10 \\
\hline $30 C^{\circ}-35 C^{\circ}$ & 24 & 8 & 12 & 8 & 0 & 9 & $55 C^{\circ}-60 C^{\circ}$ & 22 & 8 & 12 & 9 & 0 & 9 \\
\hline
\end{tabular}

Once this configuration had been selected, the different characteristics of the training carried out with back-propagation learning would be set. The training steps were fixed at ranges of 400 to 700 epochs, with an average error below $1 \%$ at the end of the training. The MLPs were trained off-line, although their performance was verified on line.

\section{Method assembly and results verification}

The method was run in the Matlab/Simulink environment. A National Instruments data acquisition card (model USB-6008 12-bit $10 \mathrm{KS} / \mathrm{s}$ Multifunction I/O) was chosen for operations at the plant. This card is automatically recognized by Matlab/Simulink. The diagram of the process is implemented in Simulink (Figure 5).

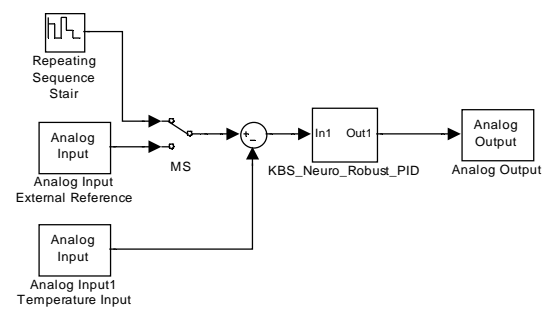

Fig. 5. System implemented in Simulink.

There are two ways of fixing the set point for the laboratory tests on the plant: the first is to generate a sequence of repeating values; the second is to set its value using an external voltage (Analog Input block diagram).

The reading of the temperature in the stove is performed by an LM35 temperature sensor conditioned with a continuous analogue output of between 0 and 10 volts. It is connected to one of the inputs of the data acquisition card in differential mode (Analog Input1). The next step is to develop the previously described controller; creating the output diagram blocks in Simulink (figure 6). 


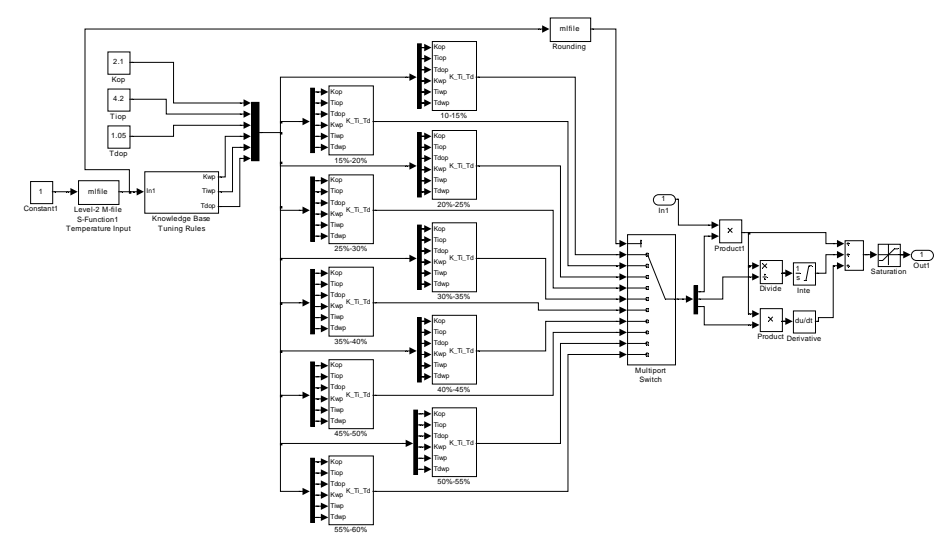

Fig. 6. Implementation of the controller.

Figure 6 shows the implementation of the parameters selection process of a PID controller, whose values are the outputs of the multiport switch element. Its inputs depend on the temperature read by the temperature sensor through analog input 2 on the data acquisition card and the parameters that are manually programmed by the operator. There are 10 blocks (Range Blocks) in the above diagram (Fig. 6), one for each range level (i.e. $25 \mathrm{C}^{\circ}$ to $30 \mathrm{C}^{\circ}$ ). Their internal layout is shown in figure 7 , where additionally, inside each Range Block there is a further block (subsystem2) that contains the scheme on the right, which is the topology of figure 2 implemented in Simulink (Matlab).
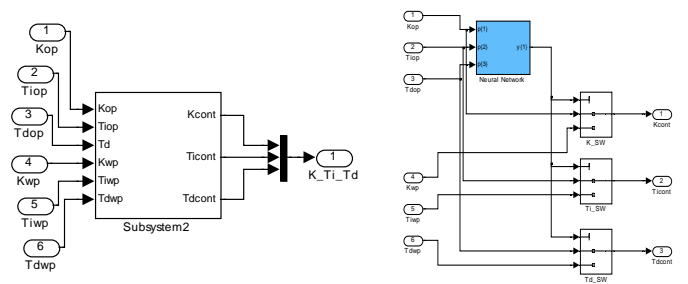

Fig. 7. Range Block internal scheme and Subsystem2 contents.

The pins In1 and Out1 (Fig 6) are the KBS_Soft_Computing_PID block pins in figure 5, which send a control signal to one of the analog outputs of the data acquisition card, to take direct action on the heating element.

In this way, the Controller will select the most appropriate parameters for the work point for which it is running. When an operator modifies the parameters, they are evaluated by the system and if there is a risk of system instability, the parameters are automatically replaced by other ones more appropriate for the operation of the work point, in such a way that they guarantee the system stability. Thus, this technique is presented as an improvement over the two previously mentioned control techniques: gain scheduling and robust control. The former has a fixed dynamic, which the operator is unable to change, while the latter can change the dynamic, but fails to assure optimal responses. In addition, it is very difficult to maintain similar specifications over all operational ranges in non-linear systems. 
The improvement may be added to other techniques that investigate leak failures in the control systems fields.

\section{Conclusions}

A new method for a Soft computing control system is presented in this study that retains the advantages offered by the conventional PID system on which it is based, as well as the possibility of applying it to non-linear systems while maintaining invariable specifications throughout the operational range. Moreover, it is set up to ensure that manual modifications to the controller parameters made by an operator to input particular specifications, for whatever reason, will at no time lead to control system instability.

The novel proposal in this research is an option to take into account in nonlinear systems that function throughout the range of operation, and that can be divided into zones with linear behaviour in which control of the PID regulator is feasible. It is therefore an alternative to different types of self-adjusting controllers. Multiple tests of the proposed Soft computing method on the laboratory plant which involved tests on the different work points yielded satisfactory results. The system is robust when an operator introduces dangerous parameter combinations in order to change operating specifications. It has to be said that the technique functioned very satisfactorily, considering the size of the stove.

\section{References}

1. Calvo-Rolle JL, Alonso-Alvarez A and Ferreiro-Garcia R "Using Knowledge Engineering in a PID Regulator in Non Linear Process Control”. Ingenieria Quimica, vol. 32, 21-28, (2007)

2. Bing C, Xiaoping L, Kefu L, Peng S and Chong L "Direct adaptive fuzzy control for nonlinear systems with time-varying delays”, Information Sciences, vol 180, no. 5, 776-792, March (2010)

3. Karimi B and Menhaj MB "Non-affine nonlinear adaptive control of decentralized large-scale systems using neural networks”, Information Sciences, vol. 180, no. 17, 3335-3347, September (2010)

4. Astrom KJ and Hagglund T “Advanced PID Control”. Pearson Education. Madrid. (2009)

5. Astrom KJ and Wittenmark B “Adaptive Control”. Addison Wesley Publis. Company. (1989)

6. Mademlis C and Kioskeridis I "Gain-Scheduling Regulator for High-Performance Position Control of Switched Reluctance Motor Drives," Industrial Electronics, IEEE Transactions on , vol.57, no.9, 2922-2931, Sept. (2010)

7. Kabbaj N, Nakkabi Y and Doncescu A “Analytical and knowledge based approaches for a bioprocess supervision”, Knowledge-Based Systems, vol. 23, no., 116-124, March (2010)

8. Bishop CM “Neural Networks for Pattern Recognition”, Oxford, (1995)

9. Baruque B and Corchado E “A weighted voting summarization of SOM ensembles”. Data Mining and Knowledge Discovery, vol. 21, no. 3, 398-426, January (2010)

10. Graupe D “Principles of artificial neural networks” 2nd ed. World Sci. Publishing Co. (2007)

11. Gaston $\mathrm{R}$ and Safonov $\mathrm{M}$ "Exact calculation of the multiloop stability margin”. IEEE Transactions on Automatic Control, 33, 156-171, (1988)

12. Canale M, Fiorio G, Malan S and Taragna M "Robust tuning of low order controllers via uncertainty model identification”. European J. of Control, Vol.5, No.2, 316-328, (1999)

13. Astrom KJ, and Hagglund T "Revisiting the Ziegler-Nichols tuning rules for PID control”, Asian Journal of Control 4 (4) 364-380. (2002)

14. Ljung L “System Identification - Theory For the User”, 2nd ed, PTR Prent. Hall, N.J., (1999) 